\title{
Latest results of 1 tonne x year Dark Matter Search with XENON1T
}

\section{Marcello Messina*t;}

New York University Abu Dhabi, Abu Dhabi, United Arab Emirates.

E-mail: marcello.messina@nyu.edu

The XENON experiment is dedicated to the search of dark matter particles interacting directly with ordinary matter, with particular focus on the Weakly Interacting Massive Particle (WIMP). The XENON experimental program, developed so far, consisted of three detectors, with the same conceptual design but increasing mass and so the sensitivity. All of them have been operated at the Gran Sasso underground laboratory in Italy, in different periods. XENON1T, the most recently operated detector and still in data-taking, is presently leading the field. The recent published results achieved the limit of the Spin Independent (SI) WIMP-nucleon cross section for WIMP masses above $6 \mathrm{GeV} / \mathrm{c}^{2}$ of $4.1 \times 10^{-47} \mathrm{~cm}^{2}$ a $90 \%$ C.L. The preparation of the next phase of the XENON project is already ongoing with the construction of the XENONnT detector, which will exploit a larger mass of $\mathrm{Xe}(\sim 8$ tonnes), and will have an improved sensitivity of one order of magnitude.

2nd World Summit on Exploring the Dark Side of the Universe 25 - 29 June, 2018

Guadeloupe Islands (France)

* Speaker.

${ }^{\dagger}$ on behalf of the XENON collaboration 


\section{Introduction}

The XENON dark matter program is devoted to search for Weakly Interacting Massive Particles (WIMPs) by detecting possible scattering of a WIMP off xenon nuclei [1]. So far three detectors have been built and operated at the Laboratori Nazionali del Gran Sasso (LNGS): XENON10 [2] on 2006 - 2007, XENON100 [3] operated in the period 2008-2016 and XENON1T operating since 2016. Beyond an increase in mass, the very successful results published by the XENON1T are based on an very careful selection of extremely low radioactivity detector components [5], the distillation of the Xe with a dedicated column [7] to reduce the contamination of radioactive ${ }^{85} \mathrm{Kr}$ to a level of few parts per trillion (ppt), and the placement of only the lowest radioactive components near the target volume. Very valuable was the collaboration with Hamamatsu to select the cleanest components to build PMTs [8] of unprecedented level of radioactive contaminants.

The XENON1T with an instrumented mass of roughy two tonnes, will have an expected sensitivity to the Spin Independent (SI) WIMP-nucleon cross section of $2 \times 10^{-47} \mathrm{~cm}^{2}$ for a WIMP mass of $50 \mathrm{GeV} / \mathrm{c}^{2}$ in $2 \mathrm{t} \cdot \mathrm{y}$ exposure.

\section{The principle of a dual-phase TPC}

Fig. 1 shows a schematic of the two-phase (liquid-gas) time projection chamber (TPC), and the signals produced by nuclear recoils are also depicted. In fact, any particle interaction in the liquid $\mathrm{Xe}(\mathrm{LXe})$ produces direct scintillation photons, labelled as S1, and ionization electrons. An electric field applied across the LXe volume drifts the ionization electrons from the interaction point towards the liquid-gas interface. Subsequently, the electrons are extracted into the gas phase, by a suited electric field $(\sim 10 \mathrm{kV} / \mathrm{cm})$, where they undergo proportional scintillation (indicated $\mathrm{S} 2$ signal) [9]. Both the S1 and S2 signals are detected by means of photomultiplier tubes (PMTs) located below the cathode (bottom array), and in the gas phase (top array). The TPC can resolve the full three-dimensional position of the interaction vertex, by using the time difference between the S1 and S2 signals which gives the depth coordinate (i.e. $z$ ) and by measuring the S2 pattern, with the top PMT array, which provides the $x$ - and $y$-coordinates. This feature is used to define a fiducial volume where the expected signal-to-background ratio is significantly enhanced, due to the excellent stopping power of the LXe. Furthermore, the ratio of the S2 over S1 signal yield is significantly different for electronic recoils (from $\gamma$ and $\beta$ scatters) from now on referred as ER, and nuclear recoils (from scatters with a neutron or WIMP candidate) named NR. This gives a powerful rejection of background interactions from electromagnetically interacting particles [10]. The irreducible background from neutrons can be estimated a priori and reduced with passive shielding, an active muon veto detector to reduce muon-spallation neutrons, and by selecting components with the lowest possible amount of radioactive contaminants.

\subsection{The XENON1T detector}

As mentioned at the beginning of the paper, the XENON1T is the third LXe TPC operated by the XENON collaboration and it is currently in data-taking. Details on the previous detectors can be found in $[2,3]$ 

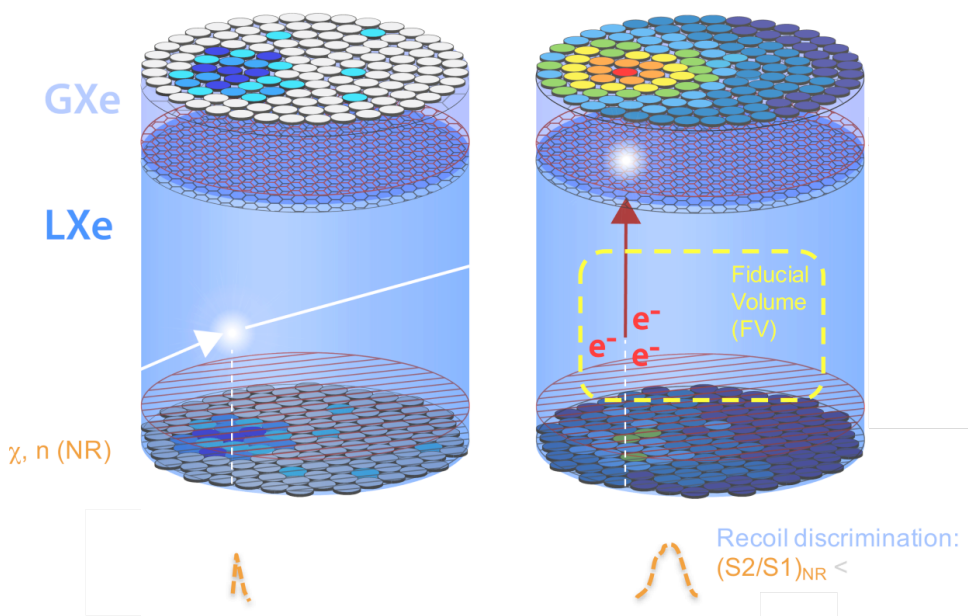

Figure 1: Schematic drawing of a generic xenon TPC and the two signals measured by it. The sketch of the $\mathrm{S} 1$ and S2 signals are also shown on the left and right image, respectively.

The core of the XENON1T ([4]) detector is the LXeTPC which is defined by a cylindrical field cage with a height of $97 \mathrm{~cm}$ and a radius of $96 \mathrm{~cm}$. The TPC is designed to contain 2 tonnes of LXe in its instrumented target. The physical volume of the instrumented target is enclosed by 24 interlocking PTFE panels that have very high reflectivity $(>0.98)$ at the LXe scintillation wavelength $(178 \mathrm{~nm})$, produced by de-excitation of excimers. The field cage is closed at the bottom by the cathode, and on top by the gate grid made with stretched wires and an etched mesh, respectively. All electrodes but the cathodes are made out of etched mesh. The field cage is also provided with field shapers set at constant potential difference to make the drift field $(116 \div 80 \mathrm{~V} / \mathrm{cm})$ as uniform as possible.

Two arrays of Hamamatsu PMTs, R11410-21, three inches in diameter, detect light in the TPC. 121 PMTs are located below the cathode, and are devoted mainly to measuring the S1 signal, while 127 PMTs are located in the gas phase, to detect primarily the S2 signal. The TPC is mounted in a double-walled 304-L-stainless-steel cryostat made of components that were screened to low level of radioactivity. Between the field cage and the wall of the cryostat there are $5 \mathrm{~cm}$ of non instrumented LXe in any direction functioning as passive shielding. The liquid level outside the TPC reaches the height of the gate, roughly.

A so called diving bell, containing the top array of PMTs, is partially immersed in the liquid. This allows the tuning of the liquid-gas interface level by means of pressure regulation. The liquid level is set in middle of $5 \mathrm{~mm}$ gap between the gate grid and an anodic grid. Such setting is very important since the $\mathrm{S} 2$ yield depends directly on it.

Due to the inherent radioactive contamination of the cryogenics system, ceramic feedthroughs, and other components, the detector is cooled remotely, and all parts with a known high radioactive contamination are installed far from the TPC itself, outside of the passive shield. The XENON1T experiment is installed underground at LNGS where a rock overburden of $3600 \mathrm{~m}$ water-equivalent, that reduces the surface muon flux by a factor of $10^{6}$, is provided. Fig. 2 shows an image of the underground installation. The increase in total mass (to 3.3 tonnes), coupled with the reduction 


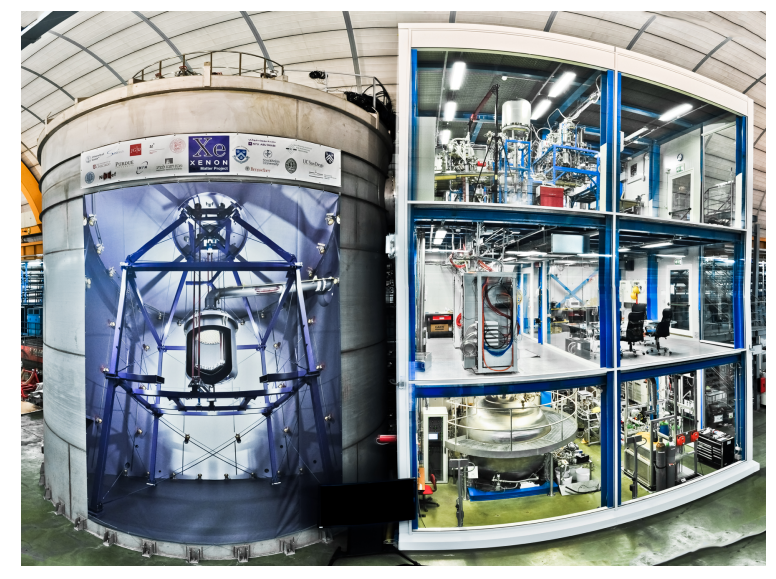

Figure 2: In the picture the water tank, which hosts the Muon Veto and the cryostat that contains the LXeTPC. The pictures show a banner showing what is inside the water thank On the right the service building with all ancillary services is also shown.

in background levels to $2 \times 10^{-4}$ events $/ \mathrm{kg} / \mathrm{day} / \mathrm{keV}$ [6], allows for two order of magnitude improvement in sensitivity with respect to XENON100. The background reduction, key to achieve the aimed at results, relies strongly on the experience gathered in the previous experiment in material selections [5] and on the partnership with Hamamatsu, that allowed to select clean materials to produce the PMTs ([8]) in use in the XENON1T detector. Equally important outcome of the experience gathered with previous detector is the distillation column installed in XENON1T. This is capable to remove the $\mathrm{Kr}$ contamination exploiting the different vapor tensions of $\mathrm{Kr}$ and $\mathrm{Xe}$. The experiment is particularly interested in removing the ${ }^{85} \mathrm{Kr}$ isotope which is beta emitter and comes naturally with the $\mathrm{Xe}$. If not removed at negligible level the $\mathrm{Kr}$ would results in an overwhelming source of background. Thus, the need of the distillation column capable to reduce the $\mathrm{Kr}$ level by a factor $6.4 \times 10^{5}([7])$, reaching a presence of ${ }^{n a t} \mathrm{Kr}$ in $\mathrm{Xe}$ of $0.65 \mathrm{ppt}$, required prior to the physics data-taking. Given the ration ${ }^{85} \mathrm{Kr} /{ }^{\text {nat }} \mathrm{Kr}=2 \cdot 10^{-11}$ the contribution of the ${ }^{85} \mathrm{Kr}$ to the ER background will be negligible with respect the most relevant source of ER background due to Rn decays, which are estimated to be $\sim 2 \times 10^{-4}$ events $/(\mathrm{kg} \times$ day $\times \mathrm{keV})$.

To stop neutrons from reaching the fiducial volume, and to tag cosmic muons (and associated neutrons), the TPC and its cryostat are immersed in $700 \mathrm{t}$ of water contained in a stainless steel tank. The tank is equipped with an active muon veto with 84 PMTs, allowing a 99\% efficiency in tagging crossing muons and $74 \%$ efficiency for showering events. The expected total background inside the fiducial volume is less than 0.01 event per $t \cdot y$.

It is worth noting that the XENON1T detector can be upgraded to a larger size detector with moderate changes. Namely, a new inner vessel in the cryostat and a TPC with larger instrumented volume will accomodate four tonnes of LXe in the fiducial volume, out of 8 tonnes of total LXe. A new purification system will be added where the Xe is purified in the liquid form to remove faster and more efficiently the electronegative impurities than the XENON1T case. Given the larger volume of Xe, a second storage system of LXe is also necessary. Ultimately a new Rn distillation column will be provided, very similar to the one used for the Kr distillation even though operated during the physics data-taking. In fact, as proved in XENON1T [7], the column is capable to keep 
the Rn, continuously emanated by all components of the TPC, in the bottom side where Xe stays in liquid phase. In this way the Rn decays mainly occur in the column so reducing significantly the background from Rn which is the most insidious one for Dark Matter search.

\subsection{Latest results of the XENON1T experiment}

Prior to any physics analysis, the response of the detector and then the energy scale need to be determined. In our case the two variables measured are S1, prompt light signal, and S2 delayed light signal generated by the ionization electrons accelerated in the gap between the gate and the anode grids. The first correction to apply is the one accounting for the effect of the electronegative impurities which deplete the ionization electrons during the drift with a time constant named "electron life-time". This effect is measured, and corrected, thanks to a diffused calibration source, ${ }^{83 m} \mathrm{Kr}$ in gaseous form with half-life of $1.83 \mathrm{~h}$. At the time of this presentation the "electron life-time" was $\simeq 650 \mu$ sec.

Furthermore, the ${ }^{83 m} \mathrm{Kr}$ is also used to correct for the non spatially uniform detector response for light and charge. The ${ }^{220} \mathrm{Rn}$ calibration sources and the ${ }^{241} \mathrm{AmBe}$ are used to study the detector response to ER and NR, respectively. The NR are also studied by using a neutron-gun installed in the water, close by the cryostat. After the calibration mentioned above, for detailed description see [11], the corrected variables cS1 and cS2 are generated. $\mathrm{cS} 2$ is actually a cS2 ${ }_{\mathrm{b}}$ since for S2 only bottom PMTs are used, given the more uniform response that they have shown. Thus, the energy scale have been studied in an interval ranging from $40 \mathrm{keV}$ to $1 \mathrm{MeV}$ where a linear correlation has been found between the energy released and a certain function of $\mathrm{cS} 1$ and $\mathrm{cS} 2 \mathrm{~b}$. Given the photon gain $g 1=0.144 \pm 0.007$ photoelectron/photon and electron gain $g 2=11.5 \pm$ 0.8 phototelectron/electron the energy calibration is $E=\left(\frac{c S 1}{g 1}+\frac{c S 2_{b}}{g 2}\right) \cdot W$, where $\mathrm{W}=13.7 \mathrm{eV}$ is the effective energy to generate a free electron or photon.

At this point two science runs (SR0, 22 November 2016 - 18 January 2017; SR1, 2 February 2017 - 8 February 2018) were jointly analyzed following a blinded data analysis approach. The total exposure is 278.8 days $\times 1.3 \mathrm{t}$ corresponding to $1 \mathrm{t} \times$ yr. The blinded approach refers to the fact that the region where the WIMP signal will be searched was blinded and the fiducial volume and the selection criteria to select the signal over background were defined prior to look at data. This should preserve the analysis from possible bias. Furthermore the data set was "salted" meaning that unknown number of events and categories were injected in the data sample to protect against possible fine-tuning of the analysis in the post unblinding phase. After it was shown that the salting was made out of two masked AmBe calibration events.

The data unblinding revealed in the region of interest (ROI), defined by $3 \leq c S 1 \leq 70$ photoelectrons (PE), $82_{-3}^{+5}($ sys $) \pm 3$ (stat) events $/(\mathrm{t} \times \mathrm{y} \times \mathrm{keV})$ which corresponds to roughly $2 \times 10^{-4}$ events $/(\mathrm{kg} \times$ day $\times \mathrm{keV})$ after efficiency correction. These results make XENON1T the experiment with the lowest background in the field of Dark Matter search. To test the WIMP signal hypothesis an un-binned profile likelihood test statistic in the space $\left(\mathrm{cS} 1, \mathrm{cS} 2_{b}, \mathrm{R}\right)$ of observables was pursued. All the uncertainties affecting the analysis have been propagated in the likelihood as nuisance parameters. Thus, the data have been found consistent with background-only hypothesis and an exclusion limit was set on the WIMP-nucleon SI cross section for WIMP masses in the range of $6-10000 \mathrm{GeV} / \mathrm{c}^{2}$. The minimum of the cross-section is $4.1 \times 10^{-47} \mathrm{~cm}^{2}$ at $30 \mathrm{GeV}$ and $90 \%$ C.L., the results are published in [11]. Fig.3 shows the best limit set in the present analysis, to- 
gether with the most recent results before XENON1T and the expected sensitivity of the next phase experiment, XENONnT.
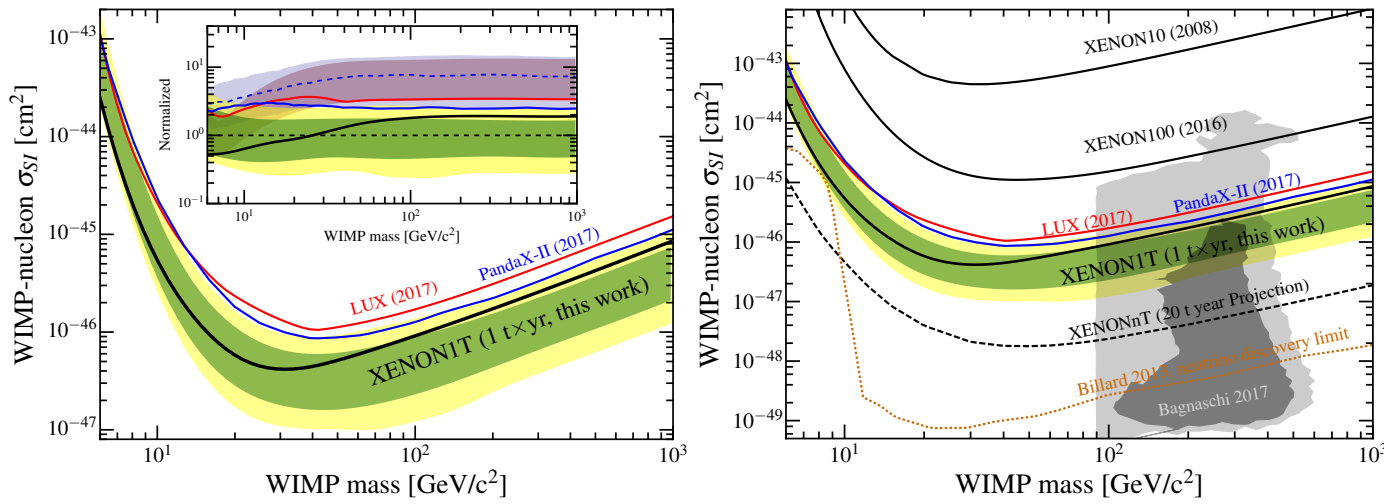

Figure 3: The left plot shows the results of this analysis and in the inset the sensitivity are also reported in comparison to previous experiments. It is worth mentioning that while the sensitivity is a factor 7 better than for other experiments the actual limit suffer of a lower improvement factor. This is due to background over-fluctuation. In the right also the projected sensitivity of the next phase experiment is shown together with the region allowed by theory and the wall of the solar neutrino background.

\section{Conclusion}

We have presented the status of the XENON dark matter program, highlighting both recent analysis results from XENON1T and prospects for the next generation detector, XENONnT. The XENON collaboration has been a leader in the field of dark matter direct detection for many years, and thanks to the XENONnT project, the collaboration looks forward to stay at the forefront of this exciting and challenging field.

\section{References}

[1] M.W. Goodman, E. Witten, Phys. Rev. D 31 3059, (1985).

[2] E. Aprile et al., Astropart. Phys. 34, 679, (2011).

[3] E. Aprile et al. Astropart. Phys. 35, 573, (2012).

[4] E. Aprile et al. Eur. Phys. J. C 77, 881, (2017).

[5] E. Aprile et al. Eur. Phys. J. C 77, 890, (2017).

[6] E. Aprile et al. JCAP 04027 (2016).

[7] E. Aprile et al. Eur. Phys. J. C 77, 275, (2017).

[8] E. Aprile et al. Eur. Phys. J. C 75, 546, (2015).

[9] B.A. Dolgoshein, V.N. Lebedenko, B.U. Rodionov,JETP Lett. 11, 513, (1970).

[10] E. Aprile et al., Phys. Rev. Lett. 97, 081302 (2006).

[11] E. Aprile et al., Phys. Rev. Lett. 121, 111302 (2018).

[12] E. Aprile et al.,Phys. Rev. Lett. 119, 181301 (2017). 